\title{
LA ACADEMIA DE INTERVENTORES DEL PROTECTORADO DE ESPAÑA EN MARRUECOS
}

\author{
Lic. D. José Luis Villanova Valero \\ Universidad de Gerona. España
}

\section{INTRODUCCIÓN}

La producción cientifica española elaborada en el último cuarto de siglo sobre Marruecos ha sido muy escasa. No obstante, a lo largo de los años noventa se observa un mayor interés por nuestro vecino meridional a raíz de la notable entrada a nuestro país de inmigrantes marroquíes, cuya presencia "va a producir la necesidad de estudios sobre el país de origen y las condiciones de partida de este colectivo" (López García, 1999, p.19).

En este contexto pueden situarse las últimas aportaciones realizadas sobre el Protectorado español, un territorio y unos habitantes que durante más de cuarenta años estuvieron bajo administración española, hasta fechas relativamente recientes. Sin duda alguna, la presencia y la acción españolas dejaron allí una impronta que aún está viva en la memoria de sus habitantes (Nogué \& Villanova, 1999).

Entre los trabajos producidos en los últimos años, y elaborados desde ópticas sensiblemente diferentes (antropología, etnografia, historia económica y política, geografia, etc.), no deja de sorprender la escasa presencia que tienen aquellos destinados a analizar el papel de las Intervenciones, "elemento esencial", "espina dorsal" o "piedra angular" -según diferentes autores- de la acción española en el Protectorado de Marruecos. Es cierto que diversos especialistas han dedicado comentarios a este importante tema, pero sólo pueden reseñarse unos pocos autores que lo hayan contemplado como eje central, o parte significativa, de alguna de sus aportaciones (por ejemplo: Torres Escobar, 1980; Teijeiro, 1987; Moga \& Bravo, 1995; Mateo Dieste, 1997a y b; o Moga, 2000).

La presente ponencia, que se enmarca en un estudio más amplio y en curso, se centra en el proceso de formación del personal interventor $y$, en concreto, en la Academia de Interventores creada en 1947, la cual fue el único centro que se ocupó de manera regular de tan trascendental tarea. 


\section{LAS INTERVENCIONES EN EL PROTECTORADO ESPAÑOL EN MARRUECOS}

La Intervención, herramienta básica en el desarrollo de la acción española en el Protectorado en Marruecos

La función interventora en el Protectorado español en Marruecos tiene su origen en el Convenio franco-español 27-11-12 que determinó el carácter de la influencia española en las Zonas Norte y Sur de Marruecos. En el artículo 1 del mismo se establecía: "Las regiones comprendidas en la Zona de influencia [...] serán administradas, con la intervención de un Alto Comisario español, por un Jalifa que el Sultán escogerá de una lista de dos candidatos presentados por el Gobierno español... Los actos de la Autoridad marroquí en la zona de influencia española serán intervenidos por el Alto Comisario y sus Agentes" (Mora \& Rodriguez, 1947, pp.15-16).

A la vista del citado convenio, diversos autores subrayaron, durante la época del Protectorado, la importancia capital de la función interventora. Por ejemplo, López Ferrer, futuro Alto Comisario, apuntaba que el "elemento esencial" de la acción española, era la intervención, la "constante intervención cerca de las autoridades indígenas" (1923, p.33); el capitán Muñoz Rocatallada consideraba a las Intervenciones "la función más importante, la más necesaria y la verdadera espina dorsal de toda organización de Protectorado" (1924, p.24); y Cordero Torres, en su obra Organización del Protectorado Español en Marruecos, al exponer su significación e importancia, indicaba que "la clave del Protectorado marroquí se encuentra en la gestión desarrollada por el sistema de Intervenciones" (1943, p.5). El sistema de Intervención constituye pues, en su opinión, "la médula de la gestión de protectorado" (op.cit., p.6).

Más recientemente, otros autores también han insistido en esta idea. Por ejemplo Morales Lezcano lo califica de "vital instrumento del colonialismo" (1986, p.120) y Hart apunta que: "El concepto de intervención fue, más que ninguna otra cosa, la piedra angular de la administración española de la zona norte" (1995, p.41).

\section{Origen y cvolución legislativa de las Intervenciones}

No es objeto de la presente ponencia analizar la enorme cantidad de disposiciones oficiales que organizaron y regularon el sistema de Intervenciones; el cual sufrió numerosas transformaciones, como consecuencia de las diferentes situaciones militares por las que pasó la Zona y de las diferentes politicas que dirigieron la acción española (Teijeiro, 1987, p.455).' Sin embargo, nos referiremos brevemente a algunas resoluciones que, por su trascendencia, es necesario comentar.

1 Para abordar este aspecto pueden consultarse, por ejemplo, los trabajos de Cordero Torres (1943, especialmente las pp.5-21) y Teijeiro (1987). 
El primer antecedente de las Intervenciones se encuentra en los Negociados de Asuntos Indigenas existentes en Ceuta y Melilla con anterioridad a la instauración del Protectorado. ${ }^{2}$ Las experiencias de estos Negociados servirán de fundamento para la organización del sistema de Intervenciones, "con las lógicas modificaciones que debieron introducirse como consecuencia del nuevo ámbito de la actuación española" (Torres Escobar, 1980, p.466).

La paulatina implantación de la presencia española en territorios próximos a Melilla comportó la creación de las Tropas de Policía Indigena, bajo el mando de Oficiales del Ejército, con la misión fundamental de mantener el orden (Real Decreto 31-12-09).

Este modelo no era original, sino que tuvo su inspiración en el francés, en los primeros Bureaux Arabes, creados con la finalidad de conocer la mentalidad "indigena" (Mateo Dieste, 1997a). ${ }^{3} \mathrm{El}$ sistema de Intervenciones que se desarrolló posteriormente -al igual que con lo sucedido con los Bureaux Arabes-, siguió el modelo del Service des Affaires Indigènes de la Zona francesa, aunque sin ser copias directas de éste (Hart, 1995, p.42).

La primera disposición que organizó administrativamente, de manera provisional, la acción española fue el Real Decreto 27-2-13. En el mismo se establecía que la Intervención política y administrativa cerca de las autoridades marroquíes sería encargada a los Cónsules en las ciudades y a los Jefes de las fuerzas militares en "las comarcas ocupadas militarmente", quienes seguirian las orientaciones dictadas por el Alto Comisario. Se concretaba así el carácter dual de la acción interventora, civil o militar según los casos.

Por Real Orden 31-7-14 se crearon las Oficinas de Asuntos Indígenas, adscritas a las Comandancias generales y calificadas como órganos de la autoridad superior en sus relaciones políticas, gubernativas, militares y judiciales con las autoridades del pais, si bien, limitándose en el aspecto administrativo y judicial, a la inspección y consejo. Se constituyeron cuadros eventuales de personal que servirian de prácticas en las Oficinas Centrales, y de los que saldria, previa selección, el personal que por su aptitud y conocimiento del árabe hubieran de cubrir las vacantes que se originaran (Cordero Torres, 1943, p.11).

El Reglamento orgánico del Protectorado, aprobado por Real Decreto 21-1-16, confirmó la existencia de un doble, o incluso triple (Torres Escobar, 1980), sistema interventor. Al Delegado de Asuntos Indígenas le correspondia ejercer "las funciones de intervención politica que el Residente general no se haya reservado especialmente" cerca de las autởidades marroquies. Pero se tenía en consideración la existencia de territorios "pacificados" $\ddot{y}$ "no pacificados", correspondiendo la Intervención en los primeros a los Cónsules, donde exis-

2 Estos negociados, colocados bajo la dependencia de los Jefes de los Estados Mayores, se encargaban de centralizar los asuntos concernientes "a los moros", territorios limitrofes y establecimientos que frecuentaban aquéllos; del despacho de asuntos tramitados por los Comandantes militares de las Plazas en sus relaciones con las cabilas limitrofes; de los asuntos de la Academia de árabe; y de estudios del pais (Real Orden 13-4-09). Su personal era designado, a propuesta de los Gobernadores militares, por el Estado Mayor dentro de una plantilla fijada previamente (Cordero Torres, 1943, pp.8-9).

3 Muestra de ello es el estudio encargado al capitán Cándido Lobera, por el Gobernador militar de Melilla, sobre los Bureaux Arabes, con la intención de proponer a la superioridad la creación de organismos análogos en las Plazas españolas (Lobera, 1905) 
tieran, y en el caso de que no los hubiera, el Interventor local sería nombrado libremente por el Gobierno, a propuesta del Alto Comisario. En los segundos, la Intervención sería ejercida por los Jefes de las Oficinas de Asuntos Indígenas.

El desastre de Annual provocó un reaplanteamiento del sistema de Intervenciones. En la exposición del Real Decreto 16-9-22 se señalaba que el atribuir la Intervención a autoridades de diverso carácter había provocado que se desarrollaran paralelamente, y sin la debida unidad, dos intervenciones políticas; por ello se expresaba el deseo de poner todos los elementos de que se hubiera de servir el Alto Comisario en el desarrollo de la política en una misma mano. Asimismo se manifestaba el principio de ir avanzando hacia el establecimiento de un régimen civil de Intervención. Sin embargo, "la realidad imperante en la zona implicaba que, con la excepción de los cónsules que limitaban su intervención a las ciudades, en el resto del territorio la misma hubiese de estar confiada a militares" (Teijeiro, 1987, p.458).

El golpe de estado de Primo de Rivera (13-9-23) y la crítica situación militar, conllevaron nuevas y constantes reorganizaciones de los organismos del Protectorado, ${ }^{4}$ de las que no escaparon las Intervenciones militares, pues las civiles, circunscritas a las ciudades, continuaron bajo el régimen citado.

Los esfuerzos reformistas de la II República en relación a la política a seguir en la Zona se encaminaron a simplificarla burocráticamente y a impregnarla de un carácter civil, tanto en las ciudades como en el campo (Morales Lezcano, 1978, p.463). No obstante, estos esfuerzos tuvieron un alcance bastante limitado debido, entre otras razones, a la ausencia de una política colonial clara, los diferentes cambios de gobierno, los obstáculos levantados por sectores decididamente colonialistas -civiles y, especialmente, militares- o la falta de personal técnico cualificado.

El Decreto 29-12-31 disponía la subordinación de las funciones militares a las civiles, colocando bajo la autoridad del Alto Comisario, a todas las autoridades del Protectorado. La Zona se dividió en regiones civiles y militares, "según la posición geográfica o la situación de las cabilas y su mayor o menor grado de pacificación". En cada una de las regiones existirfan oficinas dirigidas por un Interventor regional, quien en representación del Alto Comisario "desempeñará todas las funciones políticas, judiciales y administrativas de intervención, cerca de las Autoridades indígenas de la región", siendo además "Jefe de todos los servicio de protectorado existentes en ellas". $\mathrm{Al}$ concretar las funciones de la Delegación de Asuntos Indigenas (DAI), colocaba bajo su autoridad la función interventora ejercida en las ciudades y en las regiones civiles, mientras que la realizada en las regiones militares, dependería del Inspector de Intervención y Fuerzas jalifianas, que dependía directamente

4 Cordero Torres cita once disposiciones que, entre mayo de 1924 y diciembre de 1929, afectaron a la organización del Protectorado (1943, pp.13-15).

5 Dependientes de las oficinas "regionales" dependian las establecidas en las diferentes cabilas que constituian la región. 
del Alto Comisario. Se mantenía, a pesar de las intenciones "civilistas" que animaban el Decreto, la existencia de dos sistemas de Intervenciones. ${ }^{6}$

No es hasta el Decreto 18-12-33 cuando, por primera vez, se dictan unas normas claras para el nombramiento de interventores. Se señalaba que la función interventora "requiere que la selección del personal interventor se efectúe con toda escrupulosidad". Esta quedaba en manos del Gobierno español, a propuesta del Alto Comisario (ver, también, nota 6), pero matizando que debían tenerse en cuenta una serie de condiciones relativas a su capacitación previa, especialización profesional, etc. Estas se especificaban en el articulado, prefiriéndose a quienes habían desempeñado funciones interventoras o cargos con relación con los asuntos indígenas, en su inmensa mayor parte militares.

En consonancia con la política "civilista" de la República y dado que la pacificación no justificaba la división de la Zona en Intervenciones civiles y militares, el Decreto 26-634 las fusionó en el "Servicio de Intervención" dependiente de la DAI."

A los pocos meses se fijaron las categorias y sueldos en el Servicio de Intervenciones, las condiciones que había de reunir el personal que aspirara a desempeñar cargos en él, las pruebas que se debian superar y los requisitos necesarios para ascender con ocasión de vacantes (Decreto 21-11-34). ${ }^{8}$ La preparación de los exámenes corría a cuenta de los aspirantes, ya que no existía ningún centro de formación específico. No obstante en el Boletín Oficial de la Zona de Protectorado... se recogieron los progamas de las materias exigidas en los diferentes concursos-oposición.

El Decreto 9-5-36 dictó nuevas normas que regulaban el nombramiento del personal interventor, al considerarse que las disposiciones anteriores no habían logrado los resultados esperados, ni mejorado la efectividad del Servicio. Cordero Torres estima que tras la letra del mismo se escondía la desconfianza del Gobierno hacia muchas de las autoridades de la Zona. La libertad de movimientos que en él se otorgaban al Gobierno, fue aprovechada especialmente por las autoridades franquistas para cesar en el Servicio a interventores "no adictos" al nuevo régimen $\left(1943\right.$, p.20). ${ }^{9}$

6 Una de las novedades del Decreto es la mención a un "Cuerpo de Interventores", subordinado al Delegado de Asuntos Indigenas, que serían nombrados libremente por el Gobierno a propuesta del Alto Comisario. Dicho Cuerpo nunca llegaría a constituirse, y en el preámbulo de los Decretos 18-12-33 y 5-9-36, se plantea que "dada la índole esencialmente politica" de la función interventora y por tratarse de cargos de la confianza del Alto Comisario y por lo tanto del Gobierno español, que han de tener "plena libertad de acción para nombrar y separar a los Interventores, no es posible, ni conveniente" que éstos constituyan un Cuerpo, pues ello enervaria la indispensable amplitud de facultades con un escalafón y recargaria el Presupuesto con la inevitable secuela de derechos adquiridos $y$ excedencias.

7 En correspondencia, con este Decreto, el de 14-11-34 suprimió la función interventora de los Cónsules.

8 Esta normativa fue criticada por el Director del Heraldo de Marruecos de Larache en varios artículos publicados. Por ejemplo, el 8-12-34 censuraba la forma en que se había planteado la prueba de acceso, pues no se exjgían suficientes garantías para que se cumplieran los importantes fines que competian a los interventores, ya que el tema de exposición era elegido por los propios aspirantes.

9 A partir de 1937 desaparecen del Boletin Oficial de la Zona... las disposiciones sobre el Servicio de Intervenciones, con excepción de las referidas a las gratificaciones o a las convocatorias de cursos en la Academia de Interventores. El Servicio, sin embargo, sufrió diferentes reorganizaciones, que pueden seguirse a través de las Memorias de las Oficinas regionales y otros documentos extraoficiales (Torres Escobar, 1980, p.467). 
Cabe señalar, por último, que la Ley 8-11-41, que reorganizó tras la guerra civil la Alta Comisaría, apenas apuntó nada nuevo respecto a las Intervenciones, indicando que el Servicio, en todos sus aspectos, estaria a cargo de la DAI.

\section{Funciones y atribuciones de los interventores}

Las abundantes y complejas funciones y atribuciones de los interventores abarcaron aspectos muy amplios en numerosos campos (administrativo, político, judicial, seguridad, información, fomento, militar, etc.), que les llevaron a ser la máxima autoridad de su jurisdicción territorial. Sin entrar a analizarlas aquí de manera detallada, ${ }^{10}$ dada su importancia expondremos algunas consideraciones generales sobre las mismas.

Antes de entrar en materia, conviene apuntar que junto a las misiones y competencias recogidas en la normativa oficial, los interventores tuvieron a su cargo otras muchas no publicadas oficialmente, por interés político en unos casos o, por deficiencias en la estructura administrativa en otros (Torres Escobar, 1980, p.475).

En primer lugar, es necesario recordar que los interventores eran los encargados de llevar a cabo las iniciativas que emanaban de la superioridad, siendo "fines esenciales" de su actuación "el sostenimiento de la tranquilidad y seguridad" (Inspección general..., 1928, p.5). Estos fines debian alcanzarse a través de la vigilancia, orientación y corrección de las actuaciones de las autoridades marroquíes, y del conocimiento de las cabilas (Mateo Dieste, 1997a, p.97), es decir, efectuando una intensa labor de información.

La labor informativa, base de la seguridad del país, debía extenderse a "todo" (García Figueras, 1926, p.31) y se realizaba a través de la Mejaznia Armada," del personal de la oficina (médicos, veterinarios, escribientes, etc.) y de algunos autóctonos, a los que se pagaba por dichos servicios. En este "todo" se incluian informaciones referidas a las autoridades intervenidas, notables, nacionalistas, sospechosos, delincuentes, cofradias religiosas, extranjeros, y, hasta a los propios funcionarios de la oficina. También debian confeccionarse censos de población y de las producciones agricolas, ganaderas e industriales; recoger datos sobre las posibilidades económicas de la cabila, el estado de las vías de comunicación y sobre la posible defensa militar. Igualmente debían elaborar mapas, croquis, reseñar distancias a caballo y en coche, etc., que servian a la superioridad para acrecentar su conocimiento de la población y del territorio y poder establecer los presupuestos correspondientes y los impuestos. Por todo ello se transmitian partes informativos diarios, mensuales y trimestrales. Aprovechando estas funciones estadísticas, las oficinas realizaban una auténtica labor policíaca (Madariaga, 1999, 259).

Además de las funciones recién comentadas, las oficinas tenian diferentes competencias sobre: las Juntas Rurales Regionales y de Fracción, las Yemaas y Consejos Comunales

10 Una sintética, aunque bastante exhaustiva, relación de las mismas puede consultarse en Torres Escobar (1980, pp.471-486).

11 La Mejaznia Armada era la fuerza encargada de velar por la "tranquilidad y seguridad" en las cabilas, se organizaba según el modeloo de la Guardia Civil y erstaban a las órdenes de los interventores. 
Israelitas, las entidades administrativas locales, las asociaciones políticas y las numerosas Juntas y Comisiones que se crearon (Juntas de Beneficiencia, de Enseñanza y Regionales de Monumentos Artísticos e Históricos, Comisiones Antipalúdica y Antituberculosa). Igualmente se les confió el cobro de impuestos, la vigilancia del cumplimiento de los reglamentos y medidas relacionadas con el fomento de la colonización, de la agricultura y la ganadería, entre otras.

Para el cumplimiento de tan variadas, complejas e importantes misiones, los interventores debían tener presentes unas normas de actuación ante la población y las autoridades marroquies.

La principal misión de los interventores era el control de las autoridades para "hacer efectiva y decentemente presentable" la acción colonial (Blanco Moro, 2000, p.12) ante una población que, en su mayor parte, la rechazaba. Evidentemente, "de la favorable actitud de éstas [las autoridades] dependía el optimo encauzamiento de todo lo demás" (Moga, 2000, p.30). En este sentido se trataba de "dar vigor y personalidad en el ejercicio de sus funciones" a las autoridades (Inspección general..., 1928, p.118), realizando el interventor su misión con discreción: "Las autoridades musulmanas han de ser las que en principio manden y ejerciten la autoridad. Ellas serán las responsables del orden, las encargadas de dirigir a sus gobernados, de cobrar los impuestos (bajo nuestra inspección), y eso no será posible mas que formando primero y robusteciendo después su personalidad" (op.cit., p.119), aunque actuando, al mismo tiempo, con energía "para aplicar las normas expuestas y para exigir el cumplimiento de ellas y de las órdenes emanadas de la superioridad" (op.cit., p.121). Esta discreción y energía debían venir acompañadas de un gran respeto a la religión, la justicia fundada en ella y los usos y costumbres (Delegación de Asuntos Indígenas, 1935, pp.14-15), para no herir la sensibilidad de la población.

\section{La necesidad de formación del personal interventor}

La instauración del Protectorado originó, de manera bastante imprevista, una situación totalmente nueva en la Administración española, por tanto la formación especifica del personal destinado a la Zona se convertía en una obligación inexcusable.

En las disposiciones oficiales se subrayaba la necesidad que el personal interventor poseyera determinados conocimientos de la lengua y el pais y sus habitantes, pero hasta bastante avanzado el Protectorado no se adoptaron medidas eficaces. Por este motivo, las opiniones que subrayaban la importancia de esta formación y reclamaban su organización se repitieron de forma insistente, especialmente tras el "desastre de Annual".

El teniente Pedro Maestre, en 1923, advertía que la Intervención requería "personal competente, conocedor de la psicología del país, de su idioma y sus costumbres" y reclamaba al Gobierno la creación de "una Escuela Especial de Asuntos de Marruecos" en la que los civiles y militares que pretendieran ejercer el cargo de interventores debieran adquirir un "título de aptitud" (1923, p.183).

El mismo año, Nido resaltaba "la falta de elementos oficiales que faciliten al funcionario civil o militar ponerse en condiciones de prestar un servicio útil" y solicitaba la crea- 
ción de centros oficiales en los que se realizara "una orientación clara y definida en los estudios" (1923, p.182).

La demanda de creación de un centro especializado continuó planteándose regularmente, incluso entre personas que ocupaban altos cargos de en la Administración del Protectorado. En 1925, el Coronel Orgaz, Inspector de Intervenciones Militares, sugirió al Alto Comisario la conveniencia de "crear un organismo central de enseñanza, en donde tanto el elemento civil como el militar pudieran adquirir aquellos redimentarios conocimientos que sobre sociología y derecho musulmán en Marruecos así como sobre el idioma son indispensables a todo aquel que aspire a desempeñar algún cargo en el Protectorado", el cual serviría para crear "unidad de doctrina". ${ }^{12}$ En línea similar se manifestaron otras autoridades, como el general Sanjurjo (Revista Hispano-Africana, 1927,) o algunos interventores (Garcia Figueras, 1926).

Tambien, en ámbitos no oficiales se era consciente de esta deficiencia. La Liga Africanista, en un documento elevado al Gobierno en 1925 solicitaba preparación para el personal destinado al Protectorado (Liga Africanista Española, 1925).

Ante la parálisis de la Administración para solucionar tan grave situación, en 1927, el General inspector de Intervenciones militares propuso la elaboración de "un Manual para uso de los Interventores, donde se hallen recopiladas todas las instrucciones que se han dictado sobre el particular y algunas que pudieran añadirse para completarlas" (Archivo General de la Administración -en adelante AGA-Caja M-1241). ${ }^{13}$

Durante el periodo republicano, y a pesar de la realización de cursos de perfeccionamiento, sobre los que volveremos más adelante, continuaron elevándose peticiones insistiendo sobre estos aspectos. En 1932, el Teniente coronel de la Intervención Regional del Rif, propuso medidas para formar a los oficiales del Ejército destinados a las Intervenciones, entre las que destacaba el estudio del árabe y la preparación de conferencias, las cuales servirian de trabajos de investigación y podrían guardarse como materiales para posteriores actividades formativas (AGA Caja M-1632).

Incluso en las Cortes republicanas se llamó la atención al respecto. Martín (1973, p.133) comenta que el diputado socialista Antonio Acuña destacó el desconocimiento, por parte de "la mayor parte de los interventores", de materias tan importantes para el desarrollo de sus funciones como "usos, costumbres, derecho musulmán y los idiomas que se hablan en el Protectorado: árabe y chelja", estando por lo tanto "incapacitados para realizar

12 Sin embargo, Orgaz estimaba que el esfuerzo económico podria ser excesivo por lo que proponía la celebración de tres ciclos de conferencias al año, a cargo de funcionarios elegidos por especialidades y a los que obligatoriamente deberían asistir los Oficiales de Intervenciones. Las conferencias podrían imprimirse y constituirian una guía bibliográfica posterior para quien deseara ampliar sus conocimientos. (Una copia de este escrito puede localizarse en el Fondo "Africa" de la Biblioteca Nacional).

13 Para concretar este objetivo, propuso nombrar una ponencia, dirigida por el Coronel Segundo Jefe de la Inspección y, formada por los Comandantes de las Centrales de Larache y Melilla y el Comandante Benigno M. Portillo (AGA Caja M-1241). Sobre dicho manual ver el apartado "Obras formativas de referencia para los Interventores". 
esta labor delicadisima, lo que origina el descontento de los indígenas por la deficiencia con que se ejerce esta labor protectora".

En algunos medios de comunicación también se manifestaron opiniones respecto a este asunto. El Director del Heraldo de Marruecos de Larache, en un artículo publicado el 8-1234 , al comentar las deficiencias que presentaba la normativa del ingreso en Intervenciones, planteó la realización de prácticas en las que los futuros interventores probaran su capacidad.

Por último, durante el periodo franquista, y antes de la creación de la Academia, se reconoció la existencia de este problema y, en 1944, el Secretario General envió una carta al Delegado de Asuntos Indígenas señalando la falta personal capacitado para el desempeño de la función interventora (AGA Caja M-2375).

\section{La formación de los interventores con anterioridad a la creación de la Academia}

Si bien hasta 1947 no se fundó la Academia, éstos pudieron disponer, con anterioridad, de algunos materiales editados con el objetivo de proporcionarles algunos conocimientos indispensables ${ }^{14}$ y con diversos cursos de perfeccionamiento, aunque éstos sólamente se planearon para interventores en activo. ${ }^{15} \mathrm{~A}$ partir de la documentación consultada no se ha podido confirmar la regularidad de los cursos, habiéndose localizado únicamente información sobre tres, y especialmente de los desarrollados en 1928 y $1935 .^{16}$

Entre septiembre y diciembre de 1928, la Inspección general de Intervenciones y Fuerzas jalifianas organizó un pequeño curso de instrucción de Oficiales del Servicio de Intervenciones, "a fin de establecer de manera precisa la unidad de doctrina en el mencionado servicio" (AGA Caja M-1670).

En la convocatoria se estableció que asistirian los Jefes y Oficiales interventores e Informadores por turnos de 30 días, estableciéndose tres turnos. Su cuerpo central lo constituyeron diversas conferencias sobre Geografia e Historia de Marruecos, Derecho musulmán, Bienes Habús y Majzén, Sociología marroquí, Literatura y posesía marroquíes, Colonización, la Sanidad y la Enseñanza en Marruecos y Arqueología en la Zona.

Al finalizar, la ponencia directora dio cuenta a la superioridad de observaciones referidas a cada uno de los asistentes. Evidentemente, dichas informaciones dejaban constancia de los conocimientos y aptitudes que demostraban los interventores, las cuales, sin duda, se tuvieron posteriormente en cuenta a la hora de efectuar nombramientos, ceses y traslados.

14 En 1917, el teniente Pedro Maestre publicó un librito destinado a impartir consejos a las Oficinas destacadas de Policia Indigena. Dicha iniciativa personal, fruto de su experiencia, recibió el elogio del entonces Comandante Millán Astray, por ser una costumbre poco desarrollada en el Ejército español (Maestre, 1917, p.5). Sobre el mismo, ver el apartado "Obras formativas de referencia para los Interventores".

15 Parece ser que no se convocó ninguno para otras personas interesadas en ingresar en Intervenciones. Esto puede ser otra muestra del "monopolio" que sobre el Servicio ejercian los militares.

16 Las noticias que tenemos de la organización del curso en 1930, por parte de la Inspección general de Intervención y Fuerzas jalifianas, proceden de la publicación de algunas de las conferencias que en él se impartieron. Véase, por ejemplo, Granado (1930). 
La convocatoria del curso de 1935 ("Acuerdo del Alto Comisario", Boletín Oficial de la Zona de Protectorado..., 1935, pp. 1479-1481), fijaba su realización en el último trimestre del año y una duración de dos meses, el doble que en caso anterior, aunque sólamente se preveía la asistencia de cinco interventores designados por el Delegado de Asuntos Indigenas, siendo su Director un Interventor Regional de primera.

Sus contenidos incluían clases intensivas de Arabe vulgar o Bereber rifeño, a elección, en el Centro de Estudios Marroquies; prácticas en las Secciones de la DAI y de topografia en el campo; la redacción de trabajos semanales "sobre temas especiales", los cuales se calificarian al finalizar el curso; la comunicación de 46 conferencias, cuyos temas concretos determinaría la DAI; y visitas a distintos establecimientos oficiales -entre ellos diversas Oficinas de Intervención-, obras públicas y lugares y localidades de interés.

A pesar de la mayor duración del curso y, por tanto, del tratamiento de mayor número de temas, éste fue objeto de algunas críticas adversas. Por ejemplo, en La Gaceia de Africa se manifestaron opiniones desfavorables hacia los organizadores considerando que ignoraban lo que debia ser "un cursillo de perfeccionamicnto profesional", es decir "para profundizar en materias ya conocidas y elevar la capacidad y las aptitudes especiales que requiere el ejercicio profesional". Se valoraba que las materias tratadas en las conferencias constituían un "plan enciclopédico de materias, amontonadas sin orden ni concierto" y, por otro lado, se juzgaba negativamente que aspectos tan complejos como la sociología marroquí se abordasen en una sola conferencia. Asimismo se echaban en falta "cuestiones relacionadas con la acción económica del Protectorado, con la colonización y la agricultura, sin olvidar tampoco la enseñanza de la doctrina mutualista y cooperativa" (Equis, 1935, I y II).

\section{LA ACADEMIA DE INTERVENTORES ${ }^{17}$}

La Academia fue creada, en Tetuán, por la Orden 24-12-46, siendo Alto Comisario el general Varela y Delegado de Asuntos Indígenas el general Larrea, quien fue el encargado de organizarla.

A partir de 1947, se desarrollaron en la misma ocho Cursos de Capacitación de interventores, ${ }^{\text {is }}$ con una duración de entre cinco y nueve meses y a los que asistieron 189 alumnos. Los que probaran su aprovechamiento al concluir el periodo académico no lograban el ingreso automático en el Servicio, sino que obtenían un "Certificado de aptitud para el Servicio de Intervenciones", el cual les daba preferencia para ocupar las vacantes de Interventores de segunda clase que se produjeran, pues los nombramientos, como ya se ha señalado se producian "a dedo".

17 Las informaciones de este apartado procelen, en su mayor parte, de las convocatorias para los cursos recogidas en el Boletin Oficial de la Zona de Protectorado... y de los diferentes volúmenes que la propia Academia editó sobre los mismos.

18 El último, que estaba previsto fïnalizara el 30 de junio de 1956, no debió llevarse a cabo en su totalidad, al ponerse fïn al Protectorado en abril de aquel año y traspasar oficialmente los Servicios de la Administración española a la marroquí en julio. 
Las diferentes convocatorias ofrecieron entre 20 y 30 plazas, según las necesidades del Servicio y en las tres primeras se reservaron algunas -la mitad en la primera convocatoriapara los últimos interventores incorporados al Servicio, los cuales eran designados por el Delegado de Asuntos Indígenas. La asistencia de miembros del Servicio en activo respondía, en aquellos primeros años de funcionamiento de la Academia, a la evidente necesidad de proporcionar una formación reglada a los interventores, pues hasta el momento poco se había hecho en este sentido.

Los cursos constaban de clases teóricas y prácticas y conferencias, que se impartían en el Centro de Estudios Marroquíes de Tetuán -dependiente de la Delegación de Educación y Cultura-, al no disponer de local propio (Valderrama, s.a., p.24), así como de visitas a distintas dependencias Tras la realización de los exámenes oportunos, se iniciaba el periodo práctico, en el que todos los componentes de la Academia se trasladaban la residencia que la DAI poséa en la Alcazaba de Snada, en la cabila de Beni Itteft (Rif), para la realización de diferentes actividades prácticas $\mathrm{y}$, por último, se realizaba un viaje por toda la $Z$ Zona.

\section{Condiciones que debían reunir los aspirantes a la Academia}

La selección de los aspirantes, que debian cumplir una serie de requisitos, quedaba a la discreción del Alto Comisario, a quien se debían enviar instancias solicitando la admisión.

Las condiciones de ingreso no se modificaron excesivamente a lo largo de las convocatorias, pero si se introdujeron pequeñas modificaciones -muchas de ellas complementarias- que respondían, en parte, a la tradicional falta de previsión con que se dictaban algunas disposiciones oficiales.

Para asistir al primer curso (1947), se requería ser Oficial del Ejército de Tierra, Mar o Aire, o Licenciado en cualquier Facultad Universitaria o Escuela Especial, siendo preferidos los que hubieran prestado con anterioridad servicios en Intervenciones o en las tropas de Ifni-Sahara o que poseyeran certificados de conocimientos de árabe o bereber. En la tercera convocatoria (1950) se añadía que entre quienes cumplieran las condiciones exigidas, tendrían preferencia los Oficiales o Jefes de Complemento. Se favorecía así el ingreso de militares separados del servicio activo o de los que habian que habian realizado milicias universitarias. La siguiente (1951) limitaba la presencia de militares a los tenientes, medida bastante acertada pues el certificado que se otorgaba al finalizar los cursos capacitaba para ocupar plazas de Interventores de segunda clase, uno de los niveles más bajos en el Servicio. La exigencia de contar con personal militar dotado de una cierta experiencia, para llevar a cabo misión tan importante como la interventora, provocó que en la quinta convocatoria (1952) se requiriese a los tenientes que contaran con más de dos años de mando en tropa y que no les correspondiera el ascenso a capitán antes de 18 meses al comenzar el curso. La penúltima (1954) fue más exhaustiva que las anteriores. Los aspirantes militares sólamente podian ser tenientes del Ejército de Tierra, que no estuvieran en el primer tercio de la escala y tuvieran cumplidas las condiciones de aptitud para el ascenso. Debido a los continuos problemas que ocasionaba en el Servicio los ascensos de los tenientes, cuando eran Interventores de segunda, se comunicaba que los que ascendieran a capitán, antes de haber ascendido a Interventores de primera, podrían continuar en el mismo destino, pero cobran- 
do los emolumentos correspondientes a los Interventores de segunda. Respecto a los civiles, se concretaba que podian solicitar el ingreso los Licenciados en Derecho, Filosofia y Letras, Ciencias Naturales, Políticas y Económicas, así como los Peritos de las Escuelas Especiales que poseyeran el título de Bachiller Universitario y quienes poseyendo este último título, poseyeran el Certificado de Estudios Superiores de Interpretación otorgado por el Centro de Estudios Marroquíes de Tetuán. En la misma, también se solicitaba información sobre el estado civil de los aspirantes, y a los casados el número y edad de los hijos. Asimismo se advertía de la imposibilidad de ascenso a Interventor de primera hasta no superar los tres cursos de árabe vulgar del Centro de Estudios Marroquíes, debiendo tener en cuenta que del primero deberían aprobar el examen al finalizar el curso de capacitación, siendo esto indispensable para obtener el certificado de aptitud. En la última convocatoria (1955) quedaron excluidos los Licenciados en Ciencias Naturales y los aspirantes que estuvieran casados.

\section{Tipología del alumnado}

A pesar de que la Zona se encontraba "pacificada" hacía años y de que entre la población autóctona se contaba con numerosos "colaboradores"-muchos de ellos licenciados tras la guerra civil-, por lo que la situación fue bastante tranquila durante los años en que funcionó la Academia, la mayor parte del personal interventor continuaba perteneciendo al estamento militar, al igual que en otros muchos puestos oficiales. Numerosos miembros del Ejército, asi como gran parte de las autoridades del régimen franquista, siempre consideraron el Protectorado como un ámbito "reservado" a los militares, alegando que, para el desarrollo de la acción de España en Marruecos, poseían unas aptitudes superiores a los civiles.

Por la Academia pasaron 189 alumnos, ${ }^{19}$ de los que 150 eran militares (32 capitanes sólamente presentes en los tres primeros cursos- y 118 tenientes) y 39 civiles. Estos sólo representaron algo más del $20 \%$ del alumnado, siendo la mayor parte -algo más del $60 \%$ Licenciados en Derecho, hecho que venía favorecido por su conocimiento de algunas de las materias que debían dominar en su futuro trabajo. Sin embargo, si la presencia de civiles fue mínima durante los primeros cursos -menos del $10 \%$ en el primer curso-, en los últimos su número se incrementó de manera notable. En el séptimo los civiles constituyeron la tercera parte de los alumnos y en el último llegaron a representar el $40 \%$. Sin embargo, el "espíritu militar" no debía de ser del todo ajeno a muchos de ellos, pues, por ejemplo, los dos civiles asistentes al tercer curso habían realizado milicias universitarias (Et-Tabyi, 1950), información que seguramente constaría en las instancias de solicitud de ingreso y que la Alta Comisaría debió valorar positivamente. El incremento del elemento civil en los últimos cur-

19 Esta cifra, el grado de los militares y la titulación de los civiles varían ligeramente según las fuentes consultadas, todas ellas oficiales o editadas por organismos oficiales. Esta falta de coincidencia, y rigurosidad, vuelve a producirse al cotejar las informaciones aparecidas en el Boletin Oficial de la Zona de Protectorado de España en Marruecos y el volumen elaborado por la propia DAI, Homenaje ofrecido al Excmo. Sr. D. Tomás Garcia Figueras. I de Junio 1952-1954, en el que se informa que a partir de 1952 no se habian realizado más cursos en la Academia. Según esta obra, los Oficiales llamados al Servicio de Intervenciones, en 1953, lo habian sido por elección, permaneciendo un año en la cabila con la obligación de estudiar las asignaturas más importantes del curso y realizando los exámenes finales en Tetuán en septiembre y octubre y enero de 1954. 
sos pudo responder a una cierta tendencia de las autoridades a dotar de un cierto "civilismo" a la acción interventora, seleccionando a personal civil que podia estar mejor preparado que el militar para desarrollar determinadas funciones.

Entre los 150 militares que fueron alumnos de la Academia, se encontraban 19 capitanes y 4 tenientes procedentes del propio Servicio -cifra que representa algo más del 10\%-, en los tres primeros cursos; siendo 127 los que accedieron a la Academia sin haber tenido relación previa con el Servicio. ${ }^{20}$

\section{Reglamentos de los cursos}

El régimen de la Academia era fijado por el Alto Comisario y los programas de las asignaturas confeccionados por el Director, un Interventor Territorial designado por el Delegado de Asuntos Indígenas. Este último era también el encargado de aprobar dichos programas.

El resto de las competencias y responsabilidades del Director consistían en la organización y desarrollo de las clases -teóricas y prácticas-, de las conferencias y de los servicios necesarios para la normal marcha del curso; el entretenimiento del expediente escolar de los alumnos; la redacción de memorias e informes sobre el curso; la administración; la disciplina; la información diaria, por escrito, al Delegado de Asuntos Indigenas del desarrollo o vicisitudes de las clases; y proponer dos Interventores Comarcales al Delegado para que desempeñaran funciones de profesores permanentes, impartiendo las clases que el Director les designara.

El resto de las clases eran dadas por personas ajenas al Servicio, propuestas por la DAI al Alto Comisario. De igual forma se procedía con las personalidades encargadas de las conferencias complementarias del curso.

El control de las actividades y el régimen de la Academia eran muy rigurosos, fruto del "espíritu militar" que la rodeaba. Así, por ejemplo, los profesores debían dar cuenta diariamente por escrito al Director del desarrollo de su clase indicando materia, tema explicado, conceptuación de cada uno de los alumnos y cuantas observaciones creyeran pertinentes para el mejor funcionamiento y desarrollo de sus temas y trabajos. Mensualmente, y al terminar el periodo teórico, las prácticas y el viaje por la Zona enviaban a la Dirección conceptuaciones obtenidas por cada uno de los alumnos, haciendo constar en ellas, "amor al servicio", carácter, afición, capacida física, correctivos impuestos, trato con los naturales, etc., con el fin de dar una perfecta y exacta cuenta de las aptitudes personales de cada uno.

Sobre la censura escolar, puede subrayarse que se proponía la baja inmediata del alumno que durante dos meses obtuviera nota media de "Insuficiente" -calificación de 3 y 4 . Si la recibida en un mes era de "Malo" -calificación de 0,1 y 2-, se procedía a su baja automática.

20 Esta afirmación puede matizarse si se tiene en cuenta que en el tercer curso varios alumnos eran hijos de antiguos interventores (Et-Tabyi, 1950), hecho que debió repetirse otros años, y que comportaba una cierta relación con la Intervención 
La disciplina era rigurosísima. $Y$ así, para reflejar el comportamiento social, escolar y cívico del alumno, se les asignaba un coeficiente numérico al incorporarse a la Escuela, del que se iban sustrayendo los coeficientes parciales que correspondían a las faltas cometidas. ${ }^{21}$ La calificación mensual resultante -el coeficiente era de 10 puntos- se unía a la nota media obtenida en las clases, y la media de ambas proporcionaba la calificación mensual. A partir del segundo curso se ponderó la importancia que se concedía a las diferentes asignaturas en la formación, multiplicándose sus calificaciones por diferentes coeficientes -3 para Arabe vulgar y Sociología y Metodología interventoras; 2 para Derecho usual, Organización del Protectorado y Geografia e Historia; y 1 para Derecho musulmán y Equitación.

\section{Programas del periodo "teórico"}

Si bien a lo largo de los ocho cursos de produjeron alteraciones en los programas, a nivel general pueden reseñarse las informaciones que a continuación se exponen.

Las clases teóricas se impartían por las mañanas de lunes a sábado, en sesiones de una hora. Revisando los programas de los cursos, se observa que el Arabe vulgar era la asignatura a la que se dio más importancia. Sus clases se daban diariamente. Este hecho nada tiene de extraño si se tiene en cuenta que, a pesar de la existencia de intérpretes en las Oficinas de Intervención, era muy conveniente y necesario que los interventores dominasen la lengua de la población y de las autoridades cerca de las que debian ejercer la misión interventora. ${ }^{22}$ No obstante sorprende la ausencia de enseñanza del Bereber, o al menos no se recoge especificamente en los programas, cuando una parte importante de la población de la Zona de expresaba en dicha lengua. ${ }^{23}$

El estudio del Francés también se consideró básico, pues su conocimiento y dominio eran esenciales para los interventores destinados en cabilas fronterizas con la Zona francesa. En el primer curso también se impartieron clases de Francés diariamente aunque su presencia, posteriormente, si bien no llega a desaparecer, pierde importancia.

Otras asignaturas teóricas a las que se dedicaron una cantidad de clases notable fueron: Derecho usual y legislativo, Derecho musulmán, Sociología y Metodología interventoras y Geografia e Historia de Marruecos. Estas eran las materias, junto a las dos mencionadas

21 Por dia retrasado en un permiso, se restaba medio punto; por cada día de permiso extraordinario, dos décimas; por cada represión privada, una décima; por cada represión pública, un cuarto de punto y por falta de material escolar, un punto. Este último aspecto se omite a partir del segundo curso. Para compensar esta rigidez y estimular a los alumnos podía aumentarse el coeficiente personal a juicio del Director y de los Profesores. También, para estimularles, se les otorgaba una décima al comprobar la corrección de faltas anteriores o demostar un interés extraordinario en las actividades del curso.

22 La preocupación por que el personal interventor tuviera conocimiento de la lengua árabe se manifestó desde el primer momento en la Administración metropolitana. En las disposiciones oficiales en las que se recogian las condiciones para ejercer en las intervenciones se incluja este requisito, como ya se ha señalado. Sin embargo, dicho requisito no debió cumplirse regularmente, como se desprende de una nota (16-10-46) en la que el Jefe de la Sección Politica notificaba a la Sección $2^{\circ}$ de la DAI que el Delegado habia resuelto que todos los interventores, debían aprobar en el Centro de Estudios Marroquies el Primer Curso de Arabe Vulgar y los siguientes cursos en años sucesivos, tanto los recientes como los "antiguos" (AGA Caja M-2703)

23 Su conocimiento, como requisito para ingresar en el Servicio, llegó a equipararse al del árabe en algunas disposiciones oficiales como, por ejemplo los Decretos 21-11-34 y 9-5-36. 
anteriormente, a las que la superioridad otorgó un mayor relieve, dadas las funciones de los interventores.

Entre las asignaturas teóricas también se incluian, entre otras: Organización del Protectorado, Régimen administrativo; Información o Instituciones, Cultura y Religión musulmanas.

La clases prácticas se desarrollaban por las tardes y la duración de las mismas variaba en función de su índole. Entre ellas, se dio a la equitación la mayor consideración. Hecho que no puede extrañar si se tienen en cuenta las condiciones fisicas del territorio, el disperso hábitat de la población, las escasas vías de comunicación transitables por automóviles u otros medios motorizados, así como la necesidad de que el interventor pudiera acceder a los más aislados lugares. Asimismo se realizaban prácticas de fotografia, automóviles, topografia y técnica de la información.

Por las tardes también se efectuaban visitas a diferentes centros oficiales (Delegaciones, dependencias militares, Museos Arqueológico y de Arte Marroqú, Escuela de Artes Indigenas, etc.), infraestructuras y obras públicas finalizadas o en construcción (estación depuradora de aguas de Tetuán, presa-embalse de Dar Acoba, etc.), escuelas, Oficinas de Intervención, localidades diversas y lugares de interés histórico-artístico.

La tercera actividad desarrollada por las tardes eran las conferencias, muchas de las cuales se repitieron a lo largo de diversos cursos. Las mismas era impartidas por personas especializadas y versaban sobre temas muy diversos: geografia de la Zona; recursos naturales; antropología, usos y costumbres; enseñanza; religión musulmana; política; fuerzas militares y policiales; Servicios y organización del Protectorado; funciones y técnica interventora; acción francesa en su Zona; etc. Con las mismas se complementaban las enseñanzas de las clases teóricas. ${ }^{24}$

\section{Internado práctico en Snada y recorrido por la Zona}

Finalizado el periodo de formación teórica, se iniciaba una fase más práctica, en la que se efectuaban diferentes actividades en la Alcazaba de Snada y un recorrido por toda la Zona.

El objetivo de la estancia en Snada era la realización de prácticas en el corazón del Rif -un área bastante aislada y en la que se habia presentado una fuerte oposición a la ocupación española- y favorecer el espiritu de equipo entre los futuros interventores.

Durante esta fase de la formación, de aproximadamente un mes de duración, se impartían clases, con carácter práctico, de Arabe vulgar ${ }^{25}$, Francés, Régimen administrativo y Topografia.

24 Algunas de las conferencias fueron publicadas para que sirvieran de material de formación y perfeccionamiento a los interventores. La DAI publicó, al menos, cinco volúmenes en los que se recogieron 46 conferencias.

25 No deja de sorprender que siendo la población del Rif mayoritariamente berberófona, no se impartieran clases de esta lengua. Al menos, esto es lo que se desprende de las publicaciones de la DAI. 
Tres veces por semana, cada alumno, con un plano y una misión concreta adjudicada por el profesor correspondiente, salia con su caballo y un Mejazni, y no regresaba hasta tenerla cumplida, para lo que no había limitación de tiempo. El resto de los días se realizaban recorridos a lugares que la Dirección consideraba de interés en el proceso de formación, donde los interventores y técnicos de los diferentes Servicios de la Administración del Protectorado les exponian sus actividades (V.B.C., 1949).

Al finalizar cada semana se celebraba una reunión, presidida por el Director, a la que asistían los profesores y los alumnos y en la que se valoraban las actividades. Un resumen de los asuntos tratados y de las observaciones estimadas pertinentes por el Director se enviaba al Delegado de Asuntos Indigenas.

En este periodo práctico, también se realizaba un recorrido, de unos dos meses de duración, por toda la Zona, con la finalidad de que los alumnos obtuvieran un amplio conocimiento de la misma. Durante el viaje se visitaban Oficinas de Intervención, diferentes infraestructuras y realizaciones materiales de la acción española (granjas experimentales, aprovechamientos forestales, etc.), se organizaban encuentros con autoridades marroquies y se impartian conferencias sobre el Servicio de Intervenciones, historia o posibilidades económicas de los distintos lugares visitados, a los que se llegaba en automóvil o a caballo, según las condiciones de acceso.

\section{Obras formativas de referencia para los interventores}

La creación de la Academia y los cursos que en ella se impartieron supusieron la tardía culminación del obligado proceso de formación del personal interventor. Sin embargo, los conocimientos adquiridos durante aquellos meses, eran claramente insuficientes para ejecutar las numerosas funciones que se atribuyeron a los interventores, a pesar del intenso ritmo con que, parece ser, se desarrollaban los cursos. Por otra parte, muchos de los interventores que se encontraban en activo, en el momento de creación de la Academia, no contaban con la formación necesaria.

Ante esta situación, la superioridad aconsejaba insistentemente la lectura de materiales -muchos de los cuales habian visto la luz antes de 1947 - $^{26}$ que podían proporcionar aquellos conocimientos juzgados como imprescindibles.

Junto a las ya mencionadas conferencias de los cursos, publicadas por la DAI, los interventores disponian de las numerosas normativas y orientaciones con instrucciones concretas que se remitían regularmente desde la Delegación. Asimismo, se habían publicado diferentes materiales oficiales, como por ejemplo el Manual para el Oficial del Servicio de Intervención en Marruecos. (Inspección general de Intervención y Fuerzas jalifianas, 1928); las Orientaciones a los Interventores en la labor del Protectorado en Marruecos (Delegación de Asuntos Indígenas, 1935), redactadas éstas por un grupo de interventores bajo la dirección del general Capaz; los volúmenes de la colección "Biblioteca del

26 Su "antigüedad" no les privaba de validez, como puso de manifiesto García Figueras, en su época como Delegado de Asuntos Indigenas, al recomendar su consulta (Garcia Figueras, 1954) 
Interventor" que comenzó a editar el Servicio de Información de la DAI en 1941;27 estudios del citado Servicio sobre cabilas, cofradias o la zona francesa y su organización; o los Vademecums y trabajos publicados por las Intervenciones Territoriales y Regionales.

También contaban los interventores con aportaciones de carácter no oficial, pero de valor reconocido por la superioridad. Las mismas incluían libros (Fuente, 1920; Capdequí, 1923; Nido, 1925; García Figueras, 1926; Tarrit, 1928; Doménech, 1940; Mora Regil, 1941 o Benéitez, 1950) y artículos de prensa, especialmente en revistas como Africa, La Gaceta de Africa, Marruecos. Exponente gráfico e informativo de la acción protectora de España, Revista Hispano-Africana, etc. Algunas de las obras traducidas eran simples transcripciones al español de obras francesas (Tarrit, 1928), pero en otras se incluían aportaciones originales referidas a la Zona española (Fuente, 1920).

A partir del nombramiento de Tomás García Figueras como Delegado de Asuntos Indigenas, en abril de 1952, los interventores contarán con mayor cantidad de materiales para su formación, gracias a la preocupación del nuevo Delegado por reactivar la eficiencia del Servicio que, según sus propias palabras "había quedado en retraso" respecto a la evolución marroqui (García Figueras, 1954).

En las numerosas "cartas" que envió a los interventores, realizadas al considerar "indispensable un contacto amplio, intenso e inmediato con todos los Interventores para hacerles conocer cuáles eran las directrices del Servicio que correspondian al cumplimiento riguroso de las consignas recibidas" (op. cit.), además de insistir en las actitudes que habian de guiar la labor interventora (moralidad, sacrificio, mística, "gracia toreadora", etc.), les recuerda los materiales de los que se pueden servir en su necesaria preparación. Entre ellos pueden citarse los numerosísismos conservados en los archivos de la DAI, de los que se envió a las Oficinas Territoriales un catálogo (op. cit, p.31) y en la Biblioteca de la Delegación.

\section{BIBLIOGRAFÍA}

BENEÍTEZ CANTERO, Valentín, 1950, Sociologia marroqui: la población de la zona española del Mogreb, Tetuán, Imp. del Majzen.

BLANCO MORO, Agustín, 2000, "Estudio introductorio" in BLANCO DE IZAGA, 2000, 11-16.

BLANCO DE IZAGA, Emilio, 2000, La vivienda rifeña. Ensayo de caracteristica e interpretación con ilustraciones del autor (1930), Melilla, Ciudades Autónomas de Ceuta y Melilla. Consejerías de Cultura

27 Los dos primeros (Materne, 1941 y Bruno, 1941) fueron traducciones del francés de una conferencia presentada en el curso de instrucción preparatoria para el Service des Affaires Indigènes, en el primer caso, y de un artículo publicado en el Boletin $n^{\circ} 8$ de L'Afrique Française de 1931, en el segundo. La publicación de estas traducciones, y que fueran los dos primeros tomos de la colección, pone de manifiesto la escasa elaboración teórica que existia en la Zona española sobre temas básicos para desarrollar con éxito y efíciencia la acción de protectorado. 
BRUNO, Henry, 1941, En Busca de una Politica Indigena, Col. "Biblioteca del Interventor", Tomo II, Ceuta, Imp. Imperio.

CAPDEQUI y BRIEU, Mauricio, 1923, Apuntes sobre la zona occidental del Protectorado Marriqui Español, Madrid, San Fernando.

CORDERO TORRES, José Maria, 1942-1943, Organización del Protectorado Español en Marruecos, 2 vol., Madrid, Editora Nacional.

DELEGACIÓN DE ASUNTOS INDÍGENAS, 1935, Orientaciones a los Interventores en la labor del Protectorado en Marruecos, $1^{\circ}$ ed. de 1932, Tetuán, Tipografia Gomáriz.

DOMENECH LAFUENTE, Angel, 1940, Apuntes sobre la Zona norte del Protectorado de España en Marruecos, Ceuta, Imprenta Imperio.

EQUIS (pseud.), 1935, "El cursillo para interventores", La Gaceta de Africa, I (24-11-35) y II (26-11-35).

ET-TABYI (pseud.), 1950, "Visita de la Academia de Interventores a la Región del Kert", Marruecos. Exponente Gráfico e Informativo de la acción protectora de España, 1718 , s.p.

FUENTE, Anatolio de, 1920, Para el Oficial de Policia Indigena, Tetuán, Imp. La Papelera Africana.

GARCÍA FIGUERAS, Tomás, 1926, Temas de Protectorado, Tetuán, Imprenta de Tropas Coloniales.

—, 1954, Cartas a los Interventores, s.l., s.e., mecanografiado.

GRANADO, Manuel, 1930, Marmecos, Toledo, Imprenta, Fotograbado y Encuadernación del Colegio para Huérfanos de Infantería.

HART, David M., 1995, "Preámbulos y estudios introductorios" in MOGA \& BRAVO (eds.), 1995, 17-108.

INSPECCIÓN GENERAL DE INTERVENCIÓN Y FUERZAS JALIFIANAS, 1928, Manual para el Oficial del Servicio de Intervención en Marruecos, Madrid, Talleres del Depósito de la Guerra.

LIGA AFRICANISTA ESPAÑOLA, 1925, "Documento elevado al Gobierno por la "Liga Africanista Española"', Revista Hispano-Africana, 10, 22.

LOBERA GIRELA, Cándido, 1905, Memoria sobre la organización y funcionamiento de las Oficinas de Asuntos Arabes de Argelia y Proyecto de Bases para la creación de organismos análogos en las plazas del Norte de Africa, Melilla, Tipografia El Telegrama del Rif.

LÓPEZ GARCÍA, Bernabé, 1999, "Prólogo" in NOGUE \& VILLANOVA (eds.), 1999, 1321.

LÓPEZ FERRER, Luciano, 1923, Naturaleza juridica del Protectorado español en Marruecos, Madrid, Publicaciones de la Real Academia de Jurisprudencia y Legislación.

MADARIAGA, María Rosa de, 1999, España y el Rif. Crónica de una historia casi olvida$d a$, Melilla, Ciudad Autónoma de Melilla-U.N.E.D. Centro Asociado de Melilla. 
MAESTRE MACÍAS, Pedro, 1917, Para las Oficinas destacadas de Policía Indigena, Sevilla, Imprenta y Librería de Eulogio de las Heras.

- 1923, Divulgación y orientación del problema de Marmecos. Intervención Civil, Intervención Militar, Granada, Imprenta del diario La Publicidad.

MARTÍN, Miguel, 1973, El colonialismo español en Marruecos, Paris, Ruedo Ibérico.

MATEO DIESTE, Josep Lluís, 1997a, El "moro" entre los primitivos. El caso del Protectorado español en Marruecos, Barcelona, Fundación La Caixa.

_ $1997 \mathrm{~b}$, "Los Interventores militares en el Protectorado español de Marruecos: la codificación de lo "indigena" y los límites del gobierno indirecto", Cuadernos del Archivo Municipal de Ceuta, 11, 275-294.

MATERNE (Teniente coronel), 1941, Los Ultimos Consejos, col. "Biblioteca del Interventor", tomo I, Ceuta, Imp. Imperio.

MOGA ROMERO, Vicente, 2000, "Estudio introductorio" in BLANCO DE IZAGA, 2000, 17-102.

MOGA ROMERO, Vicente \& BRAVO NIETO, Antonio (eds.), Emilio Blanco Izaga: Coronel en el Rif. Una selección de su obra, publicada e inédita, sobre la estructura sociopolitca de los rifeños del norte de Marmecos, Melilla, Ayuntamiento de Melilla \& U.N.E.D.-Centro Asociado de Melilla.

MORA REGIL, Eugenio, 1941, Manual del Interventor en sus funciones auxiliares de la Administración de Justicia, Ceuta, Imprenta Imperio.

MORA REGIL, E. \& RODRÍGUEZ AGUILERA, C., 1947, Leyes de Marruecos, Parte I "Orgánica", Madrid, Instituto Editorial Reus.

MUÑOZ ROCATALLADA, Carlos , 1924, "Intervenciones Indigenas”, Revista de Tropas Coloniales, 1, 24-25

MORALES LEZCANO, Victor, 1978, "El Protectorado español en Marruecos bajo la II" República. (Las reformas administrativas), Actas de las Jornadas de Cultura Arabe e Islámica (1978), 1981, Madrid, Intituto Hispano-Arabe de Cultura, 457-489.

- 1986, España y el norte de Africa: el Protectorado en Marruecos (1912-1956), $2^{\mathrm{a}} \mathrm{ed}$., Madrid, U.N.E.D.

NIDO y TORRES, Manuel del, 1923, El Libro de la Mehal-la Jalifiana, Toledo, Imprenta del Colegio de María Cristina.

- 1925, Marruecos. Apuntes para el Oficial de Intervención y de Tropas Coloniales, Tetuán, Editorial Hispano Africana.

NOGUE, Joan \& VILLANOVA, José Luis (eds.), 1999, España en Marruecos (1912-1956). Discursos geográficos e intervención territorial, Lleida, Milenio.

REVISTA HISPANO-AFRICANA, 1927, "El Marruecos de hoy y el de mañana. Interesantes manifestaciones del general Sanjurjo", Revista Hispano-Africana, 32 y 33, 1-3.

TARRIT (Commandant), 1928, La Direction Génerale des Affaires Indigènes au Maroc, Casablanca, Impriméries Réunies de "La Vigie Marocaine" \& du "Petit Marocain". 
TEIJEIRO DE LA ROSA, Juan Miguel, 1987, "El Servicio de intervenciones en Marruecos", Actas del Congreso Internacional: El Estrecho de Gibraltar, Ceuta, Noviembre 1987, vol.III, Madrid, UNED \& Ayunlamiento de Ceuta, 455-466.

TORRES ESCOBAR, Francisco, 1980, "La Intervención en el Protectorado de Marruecos (1936-1956)", Revista de la Universidad Complutense, XXVIII(116), 465-486.

V.B.C. (iniciales de Valentin Beneitez Cantcro), 1949, "La Academia de Interventores", Marruecos. Exponente gráfico e informativo de la acción protectora de España, 4-5. p.s.n.

VALDERRAMA MARTÍNEZ, Fernando, s.a., El Centro de Estudios Marroquies de Tetuán, s.l., s.e. 